\title{
PET/CT for Pancreatic Malignancy: Potential and Pitfalls
}

\author{
Priyanka Jha and Bijan Bijan \\ Department of Radiology and Nuclear Medicine, University of California Davis Medical Center, Sacramento, California
}

\begin{abstract}
Pancreatic malignancy carries a poor prognosis and is the fourth leading cause of cancer-related deaths in the United States. Although conventional imaging with CT and MR remains the main imaging modality, recent times have seen an increase in the applications of PET/CT in the evaluation of pancreatic malignancy. Newer data are becoming available, highlighting the advantages, limitations, and pitfalls in PET/CT imaging of the pancreas. This article highlights the applications of PET/CT in various stages of management of pancreatic malignancy and compares these with conventional imaging with CT and MR.
\end{abstract}

Key Words: pancreatic malignancy; pancreas adenocarcinoma; PET/CT; PET/MR; pancreatic adenocarcinoma

J Nucl Med Technol 2015; 43:92-97

DOI: 10.2967/jnmt.114.145458

\section{$\mathbf{P}$}

ancreatic malignancy is a heterogeneous group of neoplasms. Eighty-five percent of malignant tumors are of exocrine origin including ductal adenocarcinoma and the rare acinar cell carcinoma $(1 \%-2 \%)$. Less common cystic neoplasms include serous and cystic pancreatic tumors (1\%$2 \%$ each) and intrapapillary mucinous neoplasms (3\%-5\%). Epithelial and mixed-differentiation tumors consist of solid pseudopapillary neoplasms $(1 \%-2 \%)$, neuroendocrine tumors $(1 \%-2 \%)$, and pancreatoblastoma $(<1 \%)(1)$.

Pancreatic adenocarcinoma has a poor prognosis, being the fourth leading cause of cancer deaths in the United States (2). Overall incidence is 12.2 cases per 100,000 persons per year, and it usually presents late, with a 5-y survival rate of $6 \%$ at the time of diagnosis $(3,4)$. Complete resection is the only cure, but only $20 \%$ of patients have potentially resectable disease at the time of presentation (4). Even patients with resectable disease enjoy a survival rate of approximately only $23 \%(2,3)$.

Contrast-enhanced CT (CECT) is the most widely used modality in the workup of pancreatic malignancy. Traditionally, CT, transabdominal ultrasound, endoscopic ultrasound, endoscopic retrograde cholangiopancreatography, MR imaging, MR cholangiopancreatography, PET, and

\footnotetext{
Received Aug. 27, 2014; revision accepted Feb. 13, 2015.

For correspondence or reprints contact: Bijan Bijan, Department of Radiology and Nuclear Medicine, University of California Davis Medical Center, 4860 Y St., Suite 3100, Sacramento, CA 95817.

E-mail: bijan.bijan@ucdmc.ucdavis.edu

Published online Apr. 9, 2015.

COPYRIGHT (c) 2015 by the Society of Nuclear Medicine and Molecular Imaging, Inc.
}

PET/CT have been applied to image pancreatic processes (Table 1). Newer data substantiating an emerging role for PET/CT have been published recently.

\section{PET/CT APPLICATION IN INITIAL MANAGEMENT OF PANCREATIC MALIGNANCY}

Most malignant tumors are hypermetabolic, compared with the normal pancreas, which demonstrates minimal to no ${ }^{18}$ F-FDG uptake (Fig. 1; Supplemental Fig. 1 [supplemental materials are available at http://tech.snmjournals. org]). Occasionally, the desmoplastic reaction associated with pancreatic adenocarcinoma may cause the lesion to appear hypometabolic (5). ${ }^{18} \mathrm{~F}-\mathrm{FDG}$ is the most widely clinically used radiotracer. Novel radiotracers, which provide greater specificity for detecting malignant processes, for example, $\sigma$-receptor ligands and $3^{\prime}$-deoxy- $3^{\prime}-{ }^{18} \mathrm{~F}-$ fluorothymidine, may be helpful in distinguishing tumor recurrence from inflammatory and fibrotic entities. This specificity is secondary to overexpression of thymidine in cancer cells (5). The degree of ${ }^{18} \mathrm{~F}-\mathrm{FDG}$ uptake is directly proportional to lesion size, particularly with most lesions larger than $1 \mathrm{~cm}$. Measuring standardized uptake values (SUVs) for tumors smaller than $1 \mathrm{~cm}$ is fraught with errors because of partial-volume averaging $(6)$.

Current recommendations include performing CT or MR imaging, particularly CECT, for evaluating patients with clinically suspected pancreatic cancer (7-9). Multiphase CT or MR is also considered appropriate for suspected pancreatic neuroendocrine tumors (10). Recent data support the role of PET/CT in the initial management for known or suspected pancreatic malignancy, particularly for characterization of lesions, staging disease, and presurgical and preradiotherapy planning.

Isodense or isointense lesions, which are occult on conventional imaging, may be detected with PET/CT (Supplemental Fig. 1) (2). The detection of such lesions on PET precedes that with CT or MR as the hypermetabolism associated with tumors can occur before anatomic alterations are evident $(11,12)$. PET/CT and CECT have been shown to have equivalent sensitivity and specificity in diagnosing pancreatic cancer $(89 \%-91 \%$ vs. $88 \%$, respectively) $(13,14)$.

PET/CT was first used to differentiate chronic pancreatitis from pancreatic malignancy. With chronic pancreatitis, the organ demonstrates diffuse but lower ${ }^{18} \mathrm{~F}$-FDG uptake versus the more focal uptake and higher maximum SUV $\left(\mathrm{SUV}_{\text {max }}\right)$ seen with tumor involvement $(1,15)$. Differentiating 
TABLE 1

Imaging Modalities to Assess for Pancreatic Malignancy in Order of Preference

\begin{tabular}{ll}
\hline \multicolumn{1}{c}{ Disease features } & \multicolumn{1}{c}{ Imaging modalities } \\
\hline Primary tumor & 1. CECT \\
& 2. Contrast-enhanced MR imaging \\
& 3. PET/CT \\
\hline \multirow{2}{*}{ Locoregional Spread } & 1. CECT \\
& 2. PET/CT \\
Vascular invasion & 1. CECT \\
& 2. Contrast-enhanced MR imaging \\
\hline Distant metastases & 1. PET/CT \\
& 2. CECT \\
\hline
\end{tabular}

mass-forming pancreatitis from pancreatic adenocarcinoma remains a diagnostic dilemma, even with CECT, especially given the fibrosis that can accompany both of these processes. Adenocarcinomas commonly obstruct the pancreatic duct, causing secondary distal pancreatitis, and chronic pancreatitis is also a well-known risk factor for adenocarcinoma, with a $20 \%$ lifetime risk by the age of $60 \mathrm{y}$, both further confounding the diagnosis (16). PET/ CT offers better characterization of mass-forming pancreatitis from adenocarcinoma than CECT $(6,13,15)$, based on the distribution and degree of ${ }^{18}$ F-FDG uptake. $\mathrm{SUV}_{\max }$ of malignant tumors is usually significantly higher than that of benign lesions, including chronic pancreatitis. Schick et al. demonstrated a sensitivity and specificity of $89 \%$ and $74 \%$, respectively, for PET/CT in differentiating mass-forming pancreatitis from malignancy (6). Other authors have demonstrated mass lesions without ${ }^{18} \mathrm{~F}-\mathrm{FDG}$ uptake at PET/CT, representing mass-forming pancreatitis (13).

Lesion size is an important consideration; lesions less than $2 \mathrm{~cm}$ in size are often ill-defined, even with CECT (17). Some studies suggest that PET/CT can better depict these lesions, with Okano et al. reporting sensitivities of $100 \%$ for PET and $40 \%$ for CECT for depicting pancreatic lesions smaller than $2 \mathrm{~cm}$ (18). Fused contrast-enhanced PET/CT has been proven to be more sensitive for tumor depiction than PET or CT alone by Lemke et al. (19).

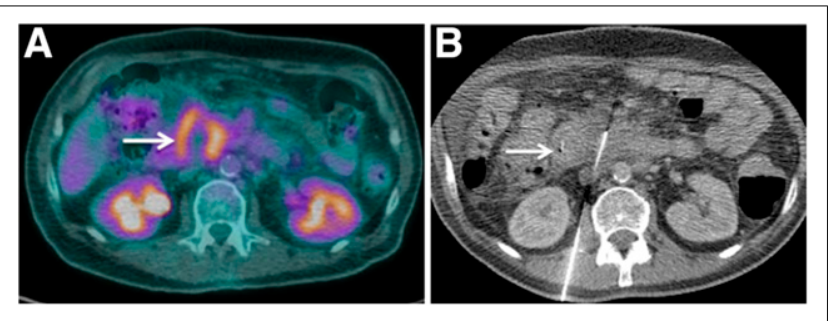

FIGURE 1. Initial staging PET/CT in a 59-y-old man with pancreatic mass of unknown etiology. (A) Axial fusion PET/CT fusion images demonstrate centrally necrotic mass with hypermetabolic peripheral rim. (B) Axial procedural CT image demonstrates targeted biopsy in progress. PET/CT was used to target hypermetabolic rim to increase diagnostic confidence. Arrows point to peripheral rim.
PET/CT plays an important role in evaluating poorly differentiated NETs and differentiating benign versus malignant cystic neoplasms such as intraductal papillary mucinous neoplasm $(20,21)$. Highly differentiated and hormonally active NETs are often ${ }^{18} \mathrm{~F}-\mathrm{FDG}-$-negative and octreotide-positive; however, the poorly differentiated NETs are mostly ${ }^{18} \mathrm{~F}-\mathrm{FDG}$-positive and octreotidenegative secondary to their high proliferative rate, hence, detectable with PET/CT (20). PET/CT was proven to be highly sensitive and accurate in distinguishing benign from malignant intraductal papillary mucinous neoplasm with mural nodules, $3 \mathrm{~mm}$ or greater in size (21). The specificity and positive predictive value were also high (100\%, using $\mathrm{SUV}_{\max }$ cutoff value of 2.3) (21).

Imaging-guided biopsies can be targeted precisely using information available from PET/CT (Fig. 1). Targeted biopsy of the most hypermetabolic portion of the tumor can lead to higher diagnostic yields, which is particularly important in heterogeneous tumors with necrosis, in tumors with significant cystic components, and with equivocal results of CT guided biopsies $(5,22)$. Specifically for pancreatic cancers, inflammatory and desmoplastic reactions associated with the tumor may be indistinguishable from the primary mass itself on conventional imaging $(5,22)$. Therefore, PET/CT can play a part in targeting the most ${ }^{18} \mathrm{~F}-\mathrm{FDG}$-avid portions of the tumor, aiding tissue diagnosis.

\section{PET/CT APPLICATION IN STAGING AND PRESURGICAL PLANNING}

The determination of resectability is the cornerstone of treatment planning for pancreatic tumors. An ideal imaging modality should be able to detect nodal and distant metastases (Figs. 2 and 3; Supplemental Fig. 2) and invasion of adjacent critical neurovascular structures including the superior mesenteric artery and vein, portal vein, celiac, hepatic, and gastroduodenal arteries. Although most of these criteria are fulfilled by CECT (23), PET has a superior performance in the initial staging of disease, mostly secondary to detection of nodal and distant metastases.

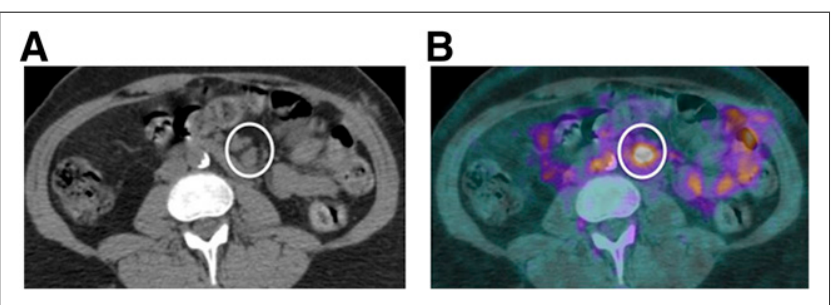

FIGURE 2. Initial staging PET/CT in a 65-y-old man with known pancreatic adenocarcinoma. (A) Axial noncontrast CT demonstrates enlarged mesenteric lymph nodes (circle). (B) Corresponding PET/CT fusion image demonstrates intense ${ }^{18} \mathrm{~F}-\mathrm{FDG}$ uptake within nodes suggestive of metastatic involvement. Physiologic uptake is present within small bowel. 


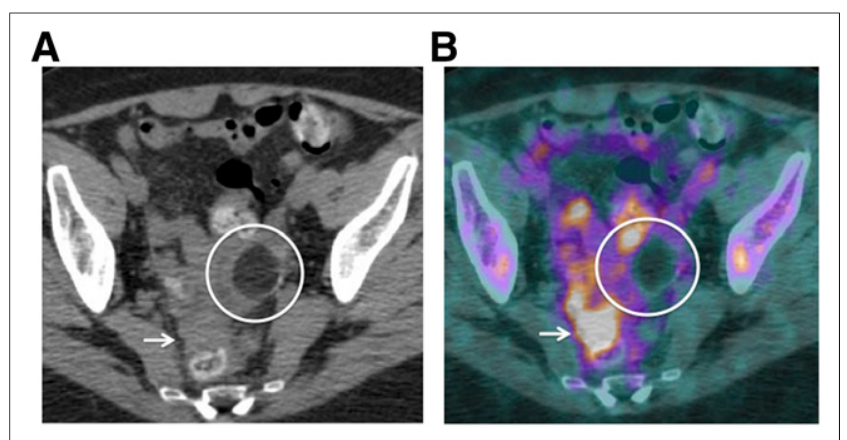

FIGURE 3. Initial staging PET/CT in a 63-y-old woman with pancreatic adenocarcinoma. (A) Noncontrast axial CT images demonstrates ill-defined peritoneal masses within pelvic culde-sac (arrow). This is another difficult location to evaluate with CT alone. (B) Corresponding PET/CT image demonstrates these masses to be intensely hypermetabolic (arrow), helpful in diagnosing peritoneal/mesenteric metastases. Incidental note is made of fat density mass (circle) within left adnexa, which does not demonstrate any ${ }^{18}$ F-FDG uptake consistent with dermoid/mature cystic teratoma.

\section{Locoregional Spread}

Assessing the local tumor spread is crucial in staging pancreatic malignancy and in deciding for potentially curative respectability. Lymph node staging is suboptimal with CT and has a $37 \%$ sensitivity and $79 \%$ specificity (Fig. 2) (24). Using traditional criteria of nodal measurements of more than $1 \mathrm{~cm}$ in the short axis doesn't adequately differentiate between benign and malignant processes. Lymph nodes smaller than $1 \mathrm{~cm}$ have been shown to be eventually malignant in nature. PET/CT allows for moderate advantage over CECT by demonstrating ${ }^{18} \mathrm{~F}$-FDG uptake within these equivocal or nonenlarged nodes (5). Asagi et al. report an accuracy of $42 \%$ for lymph node metastasis (25). However, even PET/CT may remain inadequate in cases of low tumor burden in the nodes or because of strong photon scatter from an avidly hypermetabolic primary mass, the so-called penumbra effect $(5,13,14)$.

\section{Distant Metastases}

Pancreatic malignancy most commonly metastasizes to the liver and to the peritoneum (Fig. 3; Supplemental Figs. 2 and 3). PET/CT, even without contrast, is reportedly more sensitive than conventional CT (88\%-91\% vs. 30\%-57\%, respectively) in identifying distant metastases $(13,14)$. Detecting isodense liver lesions, particularly in the setting of chemotherapy-induced steatosis, is difficult with CECT secondary to masking of these hypodense metastases over a background of hypodense liver parenchyma. Foci of hypermetabolism in these otherwise occult lesions strongly point to the presence of metastases (13). CT at best has a moderate sensitivity $(65 \%-88 \%)$ and specificity $(38 \%-63 \%)$ for detecting peritoneal metastatic implants $(26,27)$. Peritoneal metastases may be found at staging of laparoscopy in up to $7 \%$ of patients with locally unresectable pancreatic cancer without any evidence of metastasis at CECT $(26,27)$.
PET/CT was demonstrated to be more accurate and sensitive in diagnosing primary pancreatic tumor and metastases, when compared with CECT and MR imaging/ MR cholangiopancreatography (13). In this study performed by Kauhanen et al., PET was superior in the initial staging in 10 of 38 and had a reported sensitivity of $88 \%$. However, limitations persisted in diagnosing lymph node metastases (13).

PET/CT makes the most impact in the initial staging of pancreatic adenocarcinoma by detecting small metastases and distant metastases (Figs. 2 and 4; Supplemental Fig. 2). Compared with CT, transabdominal ultrasound, and endoscopic ultrasound, PET demonstrated superior sensitivity and specificity-96\% and 78\%, respectively-in diagnosing adenocarcinoma. The respective values reported in the current literature for the other 3 modalities are $91 \%$ and $56 \%$ for CT, $91 \%$ and $50 \%$ for transabdominal ultrasound, and $96 \%$ and $67 \%$ for endoscopic ultrasound (20). Barber et al. demonstrated the benefits of PET/CT over conventional imaging by detecting distant metastases and preventing unnecessary laparotomy. On the basis of PET/CT results, management was altered in $41 \%$ patients in their cohort (28), of which $33 \%$ occurred at initial staging and $43 \%$ patients at subsequent imaging, all secondary to upstaging disease (Fig. 4) (28). However, classic pitfalls such as misregistered liver metabolism mimicking lung nodules (Fig. 5) and diffuse bone marrow uptake after chemotherapy (Supplemental Fig. 4) should be realized to prevent erroneous staging of the disease.

For assessing for preoperative resectability, however, contrast-enhanced PET/CT has been found to be superior to PET alone (29). PET and PET/CT both had sensitivities of $100 \%$, however, contrast-enhanced PET/CT was superior to unenhanced PET/CT (sensitivity, 96\%; specificity, 82\%). In this study by Strobel et al., $10 \%$ of patients had unresectable tumors diagnosed intraoperatively, which were missed with all imaging modalities. These included 2 liver metastases, 1

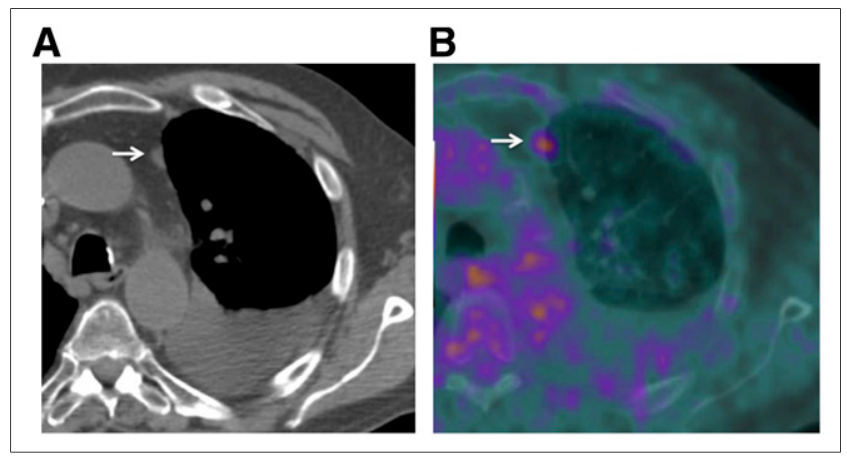

FIGURE 4. PET/CT performed for evaluating response to chemotherapy in a 69-y-old man with pancreatic adenocarcinoma. (A) Noncontrast CT shows small pleural-based mass adjacent to left lung apex (arrow). (B) Corresponding PET/CT demonstrates its hypermetabolic nature, suggestive of pleural metastasis. This was previously not identified on CECT, and presence of ${ }^{18} \mathrm{~F}$-FDG uptake led to improved identification and, hence, more accurate staging assessment with PET/CT. 


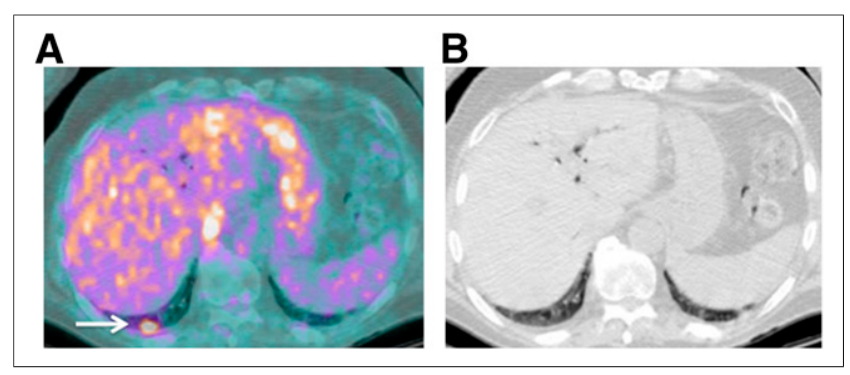

FIGURE 5. Restaging PET/CT in a 71-y-old woman with pancreatic adenocarcinoma. Hypermetabolic focus at right lung base (arrow) (A) without corresponding nodule on CT component of examination (B) represents misregistration of hepatic activity mimicking a lung nodule.

peritoneal carcinomatosis, and 2 cases of mesenteric root invasion. All cases of arterial invasion were accurately depicted by contrast-enhanced PET/CT. Contrast-enhanced PET/CT picked up more distant metastases (90\% vs. $67 \%$ vs. $43 \%$ ), peritoneal carcinomatosis ( $80 \%$ vs. $60 \%$ vs. $20 \%$ ), and arterial infiltration (100\% vs. $0 \%$ vs. 0\%) than noncontrast PET/CT and PET alone, respectively (29).

\section{PET/CT APPLICATIONS IN PRERADIOTHERAPY PLANNING}

PET/CT is helpful in estimating tumor volume and planning the conformal radiation field in candidates for radiotherapy, with improved delineation of tumor margins, compared with CT alone $(1,30)$. A single-institution experience of adding PET to $\mathrm{CT}$ resulted in an approximately $30 \%$ increase in gross tumor volume, because of incorporation of additional nodal metastases and extension of the primary volume beyond that defined with CT (31).

\section{PET/CT APPLICATIONS TOWARD PROGNOSIS, RESPONSE TO THERAPY, RECURRENCE, AND SUBSEQUENT MANAGEMENT}

Schellenberg et al. proved that SUVs obtained alone from pretreatment PET/CTs were prognostic of overall survival and progression-free survival in patients with unresectable pancreatic cancer even when controlled for age, presenting CA19-9 levels, and single versus combination chemotherapy (32). The higher the baseline $\mathrm{SUV}_{\max }$, the greater the likelihood of recurrence in the early postoperative period. $\mathrm{SUV}_{\max }$ may also be an independent predictor for overall survival in patients with locally advanced pancreatic cancer $(32,33)$. In a study performed by Sperti et al., at 1-y follow-up, none of the patients with an SUV greater than 4.0 had survived, and $75 \%$ of those with an SUV less or equal to 4.0 were still alive (34). An at least 6-wk waiting period is recommended after an intervention or radiation to limit false-positive ${ }^{18}$ F-FDG uptake secondary to inflammation from these procedures $(35,36)$.

Reduced ${ }^{18}$ F-FDG uptake may precede actual morphologic changes, allowing for increased sensitivity of PET/CT in assessing disease recurrence and response to therapy
(Supplemental Fig. 6). Pre- and postchemotherapy PET/ CT can be compared in order to estimate the degree of chemotherapeutic effect and, hence, predict survival (37). $\mathrm{PET} / \mathrm{CT}$ has been shown to improve the evaluation for cancer recurrence, particularly in patients with elevated CA19-9 levels and in those with normal or equivocal CT findings. Tissue remodeling associated with healing may hinder the assessment of treatment response on CT. Studies have noted a correlation between the variations observed in the SUV before and after radiochemotherapy and tumor necrosis. Reportedly, decreased ${ }^{18}$ F-FDG uptake after radiochemotherapy without definite response on CT may allow for subsequent surgical resection (38).

PET is more sensitive than CECT for monitoring response to chemoradiation and depicting tumor recurrence after surgery $(34,35,37,39)$. Most $(72 \%-92 \%)$ pancreatic adenocarcinomas recur locally within 2 y of surgery (34). Delineating postsurgical architectural changes from recurrent tumor is limited, even on CECT. This response may be better clarified if these findings are associated with hypermetabolism, suggesting recurrence $(6,34)$. Postoperative ${ }^{18} \mathrm{~F}$-FDG uptake in the surgical bed is expected to resolve by 3 mo after surgery, and any remaining uptake after this time is usually indicative of residual tumor or recurrence. The functional capabilities of PET make it highly $(96 \%)$ sensitive in depicting tumor recurrence, compared with $39 \%$ sensitivity for CT and MR imaging (35). For the same reason, tumor relapse is also detected earlier with PET than CT, with a higher sensitivity $(98 \%)$ and specificity $(90 \%)$ (34). Ruf et al. (38) noted a markedly superior sensitivity of PET/CT for detecting recurrence after surgery (96\%), compared with $39 \%$ with CT or MR imaging (Fig. 6; Supplemental Fig. 4) (39\%).

\section{LIMITATIONS OF PET/CT}

Both false-positive and false-negative scenarios are associated with PET/CT imaging. False-negative results can be seen with hyperglycemia, a common feature of pancreatic disease, secondary to reduced ${ }^{18} \mathrm{~F}-\mathrm{FDG}$ uptake resultant from competitive inhibition (20,38). False-negative cases may also occur in mucinous tumors (due to tissue hypocellularity) and necrotic tumors and liver and peritoneal metastases smaller than $1 \mathrm{~cm}$ $(20,38)$. False-positive cases may also result in technical problems such as misregistration of ${ }^{18}$ F-FDG uptake (Fig. 5) and attenuation overcorrection artifact (Fig. 6). Partial-volume averaging may lead to erroneous measurement of SUVs in smaller tumors and in tumors located adjacent to areas of high physiologic uptake (20). Periampullary tumors are an example of this phenomenon, because of their small size at the time of clinical presentation and proximity to ${ }^{18} \mathrm{~F}-\mathrm{FDG}$-avid bowel (20).

Pancreatic malignancy most commonly metastasizes to the liver and peritoneum (20). Identifying these lesions is key toward the most appropriate treatment. However, smaller lesions, below the size threshold of $1 \mathrm{~cm}$, are reportedly most commonly underdiagnosed (39). Small lung 


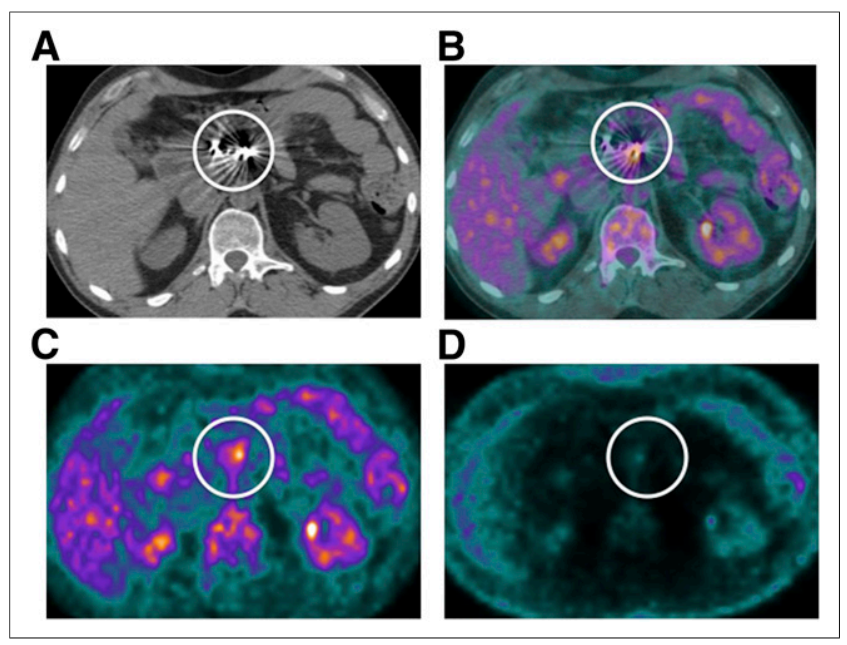

FIGURE 6. Restaging PET/CT in a 68-y-old man post Whipple's procedure with multiple surgical clips at surgical site. (A) Dense beam hardening limits evaluation for anatomic details (circle). Fused PET/CT (B) and attenuation-corrected PET (C) demonstrate hypermetabolic focus (circle) corresponding to surgical clips. (D) However, no uptake is present on nonattenuation-corrected image, suggesting that this is attenuationovercorrection artifact mimicking a lesion.

metastasis can also be overlooked on PET but detected on the CT component of the examination, supporting the complementary nature of the 2 modalities (27).

Tumor-mimicking hypermetabolic foci may occur with inflammation such as acute flare-up of chronic or autoimmune pancreatitis, recent radiation therapy, and recent surgical incision and biopsy and around common bile duct stents $(20,38)$. CECT has a higher spatial resolution than $\mathrm{PET} / \mathrm{CT}$. Along with the presence of intravenous contrast, vascular invasion is better assessed at CECT than unenhanced PET/CT (38).

\section{FUTURE DIRECTIONS}

PET radiotracers such as $3^{\prime}$-deoxy- $3^{\prime}-{ }^{18} \mathrm{~F}$-fluorothymidine are under development toward clinical application. Thymidine-based radiotracers may offer a higher specificity, because thymidine has a higher specificity for tumor cells than glucose, which is ubiquitous in the body. Tumor cells are known to overexpress $\sigma$-ligand-based thymidine receptors and hence selectively uptake $3^{\prime}-$ deoxy- $3^{\prime}-{ }^{18} \mathrm{~F}$ fluorothymidine. The role of thymidine-based tracers in pancreatic malignancy still needs to be fully evaluated, and further studies are needed.

PET/MR is an additional tool that advantageously combines the high soft-tissue resolution of MR with the functional information of PET. The lack of radiation with MR imaging is a lucrative benefit, especially for patients undergoing frequent imaging. MR may be able to detect subtle and small lesions, which may be occult on CT. Multiple sequences including T1, diffusion-weighted imaging have been evaluated for potential applications. PET/ MR imaging fusion, especially PET with T1-weighted MR imaging, compared with PET/CT, was shown by Tatsumi et al. to have improved characterization of pancreatic tumors, with better mapping and fusion image quality (40).

\section{CONCLUSION}

$\mathrm{PET} / \mathrm{CT}$ is an advantageous tool in the management of pancreatic cancer. The combination of functional and anatomic data provides a closer depiction of the biologic behavior of the tumor, compared with other conventional imaging modalities. PET/CT outperforms all imaging modalities in the detection of distant metastases, allowing for more accurate staging, despite its limitations in locoregional assessment for lymphadenopathy and vascular invasion. The limited spatial resolution of PET has been overcome by the CT component of the evaluation, and PET complements the lack of functional information on CT. The development of novel tumor-specific PET tracers such as the thymidine-based radiotracers ( $3^{\prime}$-deoxy- $3^{\prime}-{ }^{18}$ F-fluorothymidine) and $\sigma$-ligand receptors will allow for further growth of this modality.

\section{DISCLOSURE}

No potential conflict of interest relevant to this article was reported.

\section{REFERENCES}

1. Cancer facts \& figures 2015. American Cancer Society website. http://www. cancer.org/research/cancerfactsstatistics/cancerfactsfigures2015/index. Accessed May 7, 2015.

2. Dibble EH, Karantanis D, Mercier G, et al. PET/CT of cancer patients: part 1, pancreatic neoplasms. AJR. 2012;199:952-967.

3. SEER stat fact sheets: pancreas cancer. Surveillance, Epidemiology, and End Results Program website. http://seer.cancer.gov/statfacts/html/pancreas.html. Updated June 14, 2013. Accessed May 7, 2015.

4. Michl P, Pauls S, Gress TM. Evidence-based diagnosis and staging of pancreatic cancer. Best Pract Res Clin Gastroenterol. 2006;20:227-251.

5. Sahani DV, Bonaffini PA, Catalano OA, et al. State-of-the-art PET/CT of the pancreas: current role and emerging indications. Radiographics. 2012;32:1133-1158.

6. Schick V, Franzius C, Beyna T, et al. Diagnostic impact of ${ }^{18}$ F-FDG PET-CT evaluating solid pancreatic lesions versus endosonography, endoscopic retrograde cholangio-pancreatography with intraductal ultrasonography and abdominal ultrasound. Eur J Nucl Med Mol Imaging. 2008;35:1775-1785.

7. NCCN guidelines. National Comprehensive Cancer Network website. http://www. nccn.org/professionals/physician_gls/f_guidelines.asp. Accessed May 7, 2015.

8. ACR appropriateness criteria: palpable abdominal mass. American College of Radiology website. http://www.acr.org/ /media/ACR/Documents/AppCriteria/Diagnostic/ PalpableAbdominalMass.pdf. Accessed May 7, 2015.

9. ACR appropriateness criteria: painless jaundice. American College of Radiology website. http://www.acr.org/ /media/553b1108216749ecade662580febf1f0.pdf. Accessed May 7, 2015.

10. Kulke MF, Benson AB III, Bergsland E, et al. Neuroendocrine tumors: clinical practice guidelines in oncology. J Natl Compr Canc Netw. 2012;10:724-764.

11. Blodgett TM, Meltzer CC, Townsend DW. PET/CT: form and function. Radiology. 2007;242:360-385.

12. Pandit-Taskar N, Schöder H, Gonen M, et al. Clinical significance of unexplained abnormal focal FDG uptake in the abdomen during whole-body PET. AJR. 2004;183:1143-1147.

13. Kauhanen SP, Komar G, Seppanen MP, et al. A prospective diagnostic accuracy study of ${ }^{18} \mathrm{~F}$-fluorodeoxyglucose positron emission tomography/computed tomography, multidetector row computed tomography, and magnetic resonance imaging in primary diagnosis and staging of pancreatic cancer. Ann Surg. 2009;250:957-963.

14. Heinrich S, Goerres GW, Schafer M, et al. Positron emission tomography/computed tomography influences on the management of resectable pancreatic cancer and its cost-effectiveness. Ann Surg. 2005;242:235-243. 
15. van Kouwen MC, Jansen JB, van Goor H, et al. FDG-PET is able to detect pancreatic carcinoma in chronic pancreatitis. Eur J Nucl Med Mol Imaging. 2005;32:399-404.

16. Boll DT, Merkle EM. Differentiating a chronic hyperplastic mass from pancreatic cancer: a challenge remaining in multidetector CT of the pancreas. Eur Radiol. 2003;13:M42-M49.

17. Tamm EP, Loyer EM, Faria SC, et al. Retrospective analysis of dual-phase MDCT and follow-up EUS/EUS-FNA in the diagnosis of pancreatic cancer. Abdom Imaging. 2007;32:660-667.

18. Okano K, Kakinoki K, Akamoto S, et al. ${ }^{18} \mathrm{~F}$-fluorodeoxyglucose positron emission tomography in the diagnosis of small pancreatic cancer. World J Gastroenterol. 2011;17:231-235.

19. Lemke AJ, Niehues SM, Hosten N, et al. Retrospective digital image fusion of multidetector CT and ${ }^{18} \mathrm{~F}$-FDG PET: clinical value in pancreatic lesions-a prospective study with 104 patients. J Nucl Med. 2004;45:1279-1286.

20. Low G, Panu A, Millo N, et al. Multimodality imaging of neoplastic and nonneoplastic solid lesions of the pancreas. Radiographics. 2011;31:993-1015.

21. Takanami K, Hiraide T, Tsuda M, et al. Additional value of FDG PET/CT to contrast-enhanced CT in the differentiation between benign and malignant intraductal papillary mucinous neoplasms of the pancreas with mural nodules. Ann Nucl Med. 2011;25:501-510.

22. Tatli S, Gerbaudo VH, Mamede M, et al. Abdominal masses sampled at PET/CTguided percutaneous biopsy: initial experience with registration of prior PET/CT images. Radiology. 2010;256:305-311.

23. Manak E, Merkel S, Klein P, et al. Resectability of pancreatic adenocarcinoma: assessment using multidetector-row computed tomography with multiplanar reformations. Abdom Imaging. 2009;34:75-80.

24. Soriano A, Castells A, Ayuso C, et al. Preoperative staging and tumor resectability assessment of pancreatic cancer: prospective study comparing endoscopic ultrasonography, helical computed tomography, magnetic resonance imaging, and angiography. Am J Gastroenterol. 2004;99:492-501.

25. Asagi A, Ohta K, Nasu J, et al. Utility of contrast-enhanced FDG-PET/CT in the clinical management of pancreatic cancer: impact on diagnosis, staging, evaluation of treatment response, and detection of recurrence. Pancreas. 2013;42:11-19.

26. Liu RC, Traverso LW. Diagnostic laparoscopy improves staging of pancreatic cancer deemed locally unresectable by computed tomography. Surg Endosc. 2005;19:638-642.

27. Tabuchi T, Itoh K, Ohshio G, et al. Tumor staging of pancreatic adenocarcinoma using early and late-phase helical CT. AJR. 1999;173:375-380.
28. Barber TW, Kalff V, Cherk MH, et al. ${ }^{18}$ F-FDG PET/CT influences management in patients with known or suspected pancreatic cancer. Intern Med J. 2011;41: $776-783$.

29. Strobel K, Heinrich S, Bhure U, et al. Contrast-enhanced F-18-FDG PET/CT: 1stop-shop imaging for assessing the resectability of pancreatic cancer. $\mathrm{J} \mathrm{Nucl}$ Med. 2008;49:1408-1413.

30. Ford EC, Herman J, Yorke E, et al. ${ }^{18} \mathrm{~F}-\mathrm{FDG}$ PET/CT for image-guided and intensity-modulated radiotherapy. J Nucl Med. 2009;50:1655-1665.

31. Topkan E, Yavuz AA, Aydin M, et al. Comparison of CT and PET-CT based planning of radiation therapy in locally advanced pancreatic carcinoma. $J$ Exp Clin Cancer Res. 2008;27:41-47.

32. Schellenberg D, Quon A, Minn AY, et al. 18Fluorodeoxyglucose PET is prognostic of progression-free and overall survival in locally advanced pan- creas cancer treated with stereotactic radiotherapy. Int J Radiat Oncol Biol Phys. 2010;77:1420-1425.

33. Okamoto K, Koyama I, Miyazawa M, et al. Preoperative 18[F]-fluorodeoxyglucose positron emission tomography/computed tomography predicts early recurrence after pancreatic cancer resection. Int J Clin Oncol. 2011;16:39-44.

34. Sperti C, Pasquali C, Chierichetti F, et al. 18-Fluorodeoxyglucose positron emission tomography in predicting survival of patients with pancreatic carcinoma. $J$ Gastrointest Surg. 2003;7:953-959, discussion 959-960.

35. Ruf J, Lopez Hänninen E, Oettle H, et al. Detection of recurrent pancreatic cancer: comparison of FDG- PET with CT/MRI. Pancreatology. 2005;5:266272.

36. Blake MA, Singh A, Setty BN, et al. Pearls and pitfalls in interpretation of abdominal and pelvic PET-CT. Radiographics. 2006;26:1335-1353.

37. Cameron K, Golan S, Simpson W, et al. Recurrent pancreatic carcinoma and cholangiocarcinoma: ${ }^{18} \mathrm{~F}$-fluorodeoxyglucose positron emission tomography/ computed tomography (PET/CT). Abdom Imaging. 2011;36:463-471.

38. Pery C, Meurette G, Ansquer C, et al. Role and limitations of ${ }^{18}$ F-FDG positron emission tomography (PET) in the management of patients with pancreatic lesions. Gastroenterol Clin Biol. 2010;34:465-474.

39. Yoshioka M, Sato T, Furuya T, et al. Role of positron emission tomography with 2-deoxy-2-[ $\left[{ }^{18} \mathrm{~F}\right]$ fluoro-d-glucose in evaluating the effects of arterial infusion chemotherapy and radiotherapy on pancreatic cancer. J Gastroenterol. 2004;39: 50-55.

40. Tatsumi M, Isohashi $\mathrm{K}$, Onishi $\mathrm{H}$, et al. ${ }^{18} \mathrm{~F}-\mathrm{FDG}$ PET/MRI fusion in characterizing pancreatic tumors: comparison to PET/CT. Int J Clin Oncol. 2011;16:408-415. 